\title{
DIVISION III / SERVICE MINOR PLANET CENTER
}

\section{DIRECTOR \\ ASSOCIATE DIRECTOR DIRECTOR EMERITUS}

\author{
Timothy B. Spahr \\ Gareth V. Williams \\ Brian G. Marsden
}

\section{TRIENNIAL REPORT 2009-2012}

\section{Introduction}

The activity of the Minor Planet Center continued generally to increase during the triennium. This report covers the period 2008 July 1 to 2011 October 12, and the phrase "during the triennium" in this report should be understood to mean this period.

During the triennium, the total number of observations in the MPC's files increased by 56\%, from 55.4 million to 86.4 million. The next-generation Pan-STARRS (Panoramic Survey Telescope and Rapid Response System) program began to produce results, and the existing Catalina Sky Survey, Mount Lemmon Survey, Siding Spring Survey and Lincoln (Laboratory) Near-Earth Asteroid Research (LINEAR) programs continued to be very productive. In addition, the Wide-field Infrared Survey Explorer (WISE) spacecraft produced two million observations from low Earth orbit.

The number of numbered minor planets increased by $60 \%$, from 189005 to 301841 , during the triennium. The numbering of a minor planet should signify that the object has been sufficiently well observed to ensure that it is unlikely to be lost in the foreseeable future. The numbered minor planets represented $46 \%$ of the orbits in the MPC files in mid-2008 and 53\% in 2011 October.

\section{Publications and archiving}

The permanent archiving of data continues to be done on a monthly basis, coinciding with the publication of the Minor Planet Circulars and the Minor Planet Circulars Orbit Supplement. As the traditional publication of the MPC, dating back to 1947, the former is a summary of MPC activity, the 13362 pages published during the triennium brings the total to 76678. The Orbit Supplement, first published in 2000, gives full details on the new numberings and the new identifications, the 68138 pages published during the triennium brings the total to 208366. The Minor Planet Circulars Observation Supplement, listing the bulk of the minor-planet observations, began publication in 1997 and are issued weekly, except around the time of Minor Planet Circulars production. The 142970 pages published during the triennium brings the total to 394976. All three permanent publications are now published only in machine-readable form, via PDFs downloadable from the MPC's website.

In addition, the Minor Planet Electronic Circulars (MPECs), first issued in 1993, provide immediate information for newly-designated NEOs, TNOs and comets. A total of 6167 MPECs were issued through the preparation of the 2011 October 12 batch of MPCs, bringing the total issued since inception to 22222. The 'Daily Orbit Update' (DOU) MPECs, prepared entirely automatically each night, tabulates all the orbits computed and identifications found at the MPC during the previous 24 hours. Each DOU issue also includes continuing observations of all NEOs, the automatic preparation precluding 
the crediting of the observers in a reliable fashion. The DOU issue is consistent with the intention that the MPECs are a temporary publication, for as long as further observations are made, orbit computations will always be improved.

The compromise of a staff member's work account by an external hacker on 2010 February 12 caused a major disruption. Internal access to the MPC's website, then hosted by the Center for Astrophysics Computation Facility, was cut off immediately as a security measure, meaning that no updates to the site were possible and that no circulars could be mailed out. A massive effort during that day meant that the external disruption was minimized: the DOU MPEC the next morning was issued on schedule (although there was a delay in its e-mail distribution) and issuance of discovery MPECs resumed the next day. Access to the NEOCP was possible without interruption thanks to mirror pages hosted on the script server machine, which continued to be updated throughout the crisis. It took three days to get the MPC's website restarted on an external host and some weeks to copy over the entire site. The MPC website continued to be hosted solely on the external site until 2011 January 19, when the new MPC-hosted website came on line. The two sites ran in parallel until 2011 June 7 , when the external site was shut down. The new website has an interface to the MPC Database, a MySQL database that can be queried on-line, allowing user-definable access to most of the public data available from the MPC.

The MPC also hosts the Light Curve Database (LCDB), a repository of minor-planet light-curve observation files, on behalf of an outside group. The LCDB contains over a million individual observations in 12641 separate data files representing 1802 different minor planets. The LCDB can be queried via the MPC website and observers can upload new data sets through the DB interface.

The "NEOCP Blog" and "Daily Minor Planet Center" were brought on-line during the triennium to serve as conduits between the MPC and the observers. The "NEOCP Blog" deals exclusively with the NEOCP and allows observers to send reports on what they are planning to observe and what they actually do observe. Posts on the blog are of a transitory nature. The "Daily Minor Planet Center" is intended for longer articles of a permanent nature, written on a variety of topics by the MPC staff.

\section{Near-Earth objects}

Prior to the preparation of an MPEC documenting a discovery, alerts to possible NEOs and comets are issued on "The NEO Confirmation Page". This page has existed since 1996 and most new objects are posted to the page automatically, as are updates to the predictions as further observations are obtained. The NEOCP underwent a major upgrade in 2011 April, allowing observers more control over the selection of objects that are returned.

During the triennium, 2763 separate NEOs were discovered, and of these 299 are considered potentially hazardous asteroids (PHAs). These objects have minimum orbital intersection distances with Earth less than 0.05 AU and absolute magnitude $\mathrm{H}<22$. More than $97 \%$ of the discoveries are made by professional astronomers (over $99 \%$ of which were US-based or US-funded surveys), but there is still room for amateurs to contribute to the NEO effort by performing astrometric follow-up of NEOs and NEO candidates.

The discovery of $2008 \mathrm{TC}_{3}$ in 2008 October led to the first confirmed short-term prediction of an Earth impact. Small fragments of the object survived passage through the atmosphere to fall as meteorites in the Sudanese desert. As a direct result of this event, the MPC implemented an internal/external alert system, which sends alerts about potential very close Earth approaches to MPC staff members and, if an external alert is triggered, to a number of JPL scientists and NASA's NEO Observations Program Executive. Once an external alert is sent out, the object is not announced until a review of the situation is completed. The external alert system has been triggered about six times during the triennium. 
Confusion of high-altitude artificial satellites as new NEOs continued to be a problem. The MPC tried to keep track of these objects using data from a variety of sources, but a number of NEO candidates that turned out to be untracked high-altitude objects were observed for only a short while before being lost again.

\section{Comets \& distant objects}

Some of the NEO candidates appearing on the NEOCP turned out to be comets. The MPC worked in cooperation with the Central Bureau for Astronomical Telegrams (a service of Division XII/Commission 6) in the announcement of new comets. Routine astrometric follow-up of comets was handled by the MPC, with temporary publication of new observations and orbits generally weekly on special MPEC $s$ and permanent archiving in the monthly Minor Planet Circulars. Almost 183000 comet observations were published during the triennium, bringing the total in the MPC archive to 595000.

Although the last triennium saw the discovery of only 112 new 'distant objects' (centaurs and transneptunian objects), bringing the total number known to 1549 , some $52 \%$ of recent discoveries have already been observed at more than one opposition. Of the total known population (including the transneptunian dwarf planets) 234 have been numbered, while $39 \%$ have been seen at only one opposition.

\section{Outer satellites of the giant planets}

Because of their potential confusion with minor planets, the MPC continued to catalogue observations and to compute orbits for the outer satellites of the giant planets. Although many astrometric observations of known objects were received during the triennium (and published in the Minor Planet Circulars), there was little discovery activity. Two new satellites of Jupiter were reported from the 2010 opposition and both objects were recovered in 2011. One Jovian satellite observed at the 2003 opposition was rediscovered in 2009 and subsequently numbered and named as Jupiter L (Herse).

\section{Personnel}

Director Emeritus B. G. Marsden died on 2010 November 18, having continued working on MPC matters up until about a week before his death, with his last $M P E C$ being issued late on November 10 and his last emails being sent on November 12. Former Associate Director C. M. Bardwell died on 2010 May 14. He began working at the MPC in 1957 and although he retired at the end of 1989, he continued to work for many years thereafter.

Director T. B. Spahr's workload continued to include normal (and abnormal) administrative duties. He worked principally with NEOs and made great inroads in extracting useful information from the file of One-Night Stand observations. He also had external teaching responsibilities. Associate Director, G. V. Williams, continued to be responsible for the bulk of the processing code, the processing of the comet and natural satellite observations, and the lion's share of the preparation of the permanent MPC publications. In addition, he served as secretary of (and MPC representative on) the Committee on Small-Body Nomenclature and as the MPC-CSBN representative on the Working Group for Planetary System Nomenclature. J. L. Galache was in charge of maintaining the MPC Blog, the Near-Earth Object Confirmation Page Blog, and bringing MPC communication front and center with modern social media technology. S. Keys wrote specialty software for the MPC, principally the MPC's NEO probability code, DIGEST2. This program, which is available for download to outside users, automatically selects from incoming astrometric data those objects that have the highest chance of being NEOs, allowing them to be posted on the NEO Confirmation Page. M. Rudenko, now moved to Harvard, was responsible for the setup and maintenance of the MPC's Linux systems, both the internal computational systems and the external web servers, and the databases. 